\title{
CD155 Overexpression Correlates With Poor Prognosis in Primary Small Cell Carcinoma of the Esophagus
}

\section{OPEN ACCESS}

Edited by:

Gaurav Malviya,

University of Glasgow,

United Kingdom

Reviewed by:

Lutao Du,

Second Hospital of Shandong

University, China

Hidemi Toyoda,

Mie University, Japan

Cinzia Fionda,

Sapienza University of Rome, Italy

*Correspondence:

Xiangjiao Meng mengxiangjiao@126.com

Jinming Yu

sdyujinming@163.com

Specialty section:

This article was submitted to Molecular Diagnostics and

Therapeutics,

a section of the journal

Frontiers in Molecular Biosciences

Received: 01 October 2020 Accepted: 07 December 2020

Published: 07 January 2021

Citation:

Zhao K, Ma L, Feng L, Huang Z, Meng $X$ and $Y u J$ (2021) CD155 Overexpression Correlates With Poor

Prognosis in Primary Small Cell Carcinoma of the Esophagus.

Front. Mol. Biosci. 7:608404.

doi: 10.3389/fmolb.2020.608404

\begin{abstract}
Kaikai Zhao ${ }^{1,2}$, Lin $\mathrm{Ma}^{3}$, Lei Feng ${ }^{4}$, Zhaoqin Huang ${ }^{5}$, Xiangjiao Meng ${ }^{6 *}$ and Jinming $\mathrm{Yu}^{7 *}$
${ }^{1}$ Department of Radiation Oncology, The First Affiliated Hospital of China Medical University, Shenyang, China, ${ }^{2}$ Department of Radiation Oncology, Yantai Affiliated Hospital of Binzhou Medical University, Yantai, China, ${ }^{3}$ Cheeloo College of Medicine, Shandong University, Jinan, China, ${ }^{4}$ Department of Radiation Oncology, Shandong Cancer Hospital and Institute, Shandong First Medical University and Shandong Academy of Medical Sciences, Jinan, China, ${ }^{5}$ Department of Radiology, Shandong Provincial Hospital Affiliated to Shandong First Medical University, Jinan, China, ${ }^{6}$ Department of Radiation Oncology, Shandong Cancer Hospital and Institute, Shandong First Medical University and Shandong Academy of Medical Sciences, Jinan, China, ${ }^{7}$ Department of Radiation Oncology, The First Affiliated Hospital of China Medical University, Shenyang, China
\end{abstract}

CD155/TIGIT overexpression has been detected in various human malignancies; however, its expression status in primary small cell carcinoma of the esophagus (PSCCE) and its prognostic significance remain unclear. In this study, we aimed to explore the expression and prognostic value of CD155 and TIGIT in PSCCE. We detected CD155 and TIGIT expression in 114 cases of PSCCE using immunohistochemistry $(\mathrm{IHC})$ and evaluated their relationship with the clinicopathological characteristics and survival of the patients. Survival analyses were performed using the Kaplan-Meier method and Cox proportional hazards model. Nomogram performance was assessed via the concordance index (C-index) and calibration plots. Decision curve analysis (DCA) was performed to evaluate the net benefit of the nomogram. We found that CD155 and TIGIT were overexpressed in PSCCE tissues, CD155 expression correlated positively with TIGIT $(p<0.001)$ and was significantly associated with tumor size, T stage, distant metastasis, TNM stage, and Ki-67 score. TIGIT expression was also significantly associated with T stage, distant metastasis, and TNM stage. Patients with high CD155 and TIGIT expression had a significantly shorter overall survival (OS) and progression-free survival (PFS), while the multivariate model showed that CD155 expression and the therapeutic strategy are independent prognostic factors for PSCCE. In the validation step, OS was shown to be well-calibrated (C-index $=0.724)$, and a satisfactory clinical utility was proven by DCA. In conclusion, our findings revealed that CD155 and TIGIT are highly expressed in patients with PSCCE and are associated with shorter OS and PFS, supporting their role as prognostic biomarker.

Keywords: primary small cell carcinoma of the esophagus, immunohistochemistry, CD155, TIGIT, prognosis

\section{INTRODUCTION}

Primary small cell carcinoma of the esophagus (PSCCE) is a rare but aggressive disease that is associated with early metastasis and poor prognosis (Lv et al., 2008; Chen et al., 2016; Wong et al., 2017; Xu et al., 2017). Patients with PSCCE are usually treated by surgical resection, chemotherapy (CT), and radiotherapy (RT) either alone or in combination (Meng et al., 2013; Wang et al., 2015; Zou et al., 2016; Xu et al., 2017). Despite progress in diagnostic 
technologies and treatment strategies, many patients with PSCCE are diagnosed at an advanced stage, and the median overall survival (OS) is only 12.5-16.7 months with traditional therapies (Xu et al., 1989; Lv et al., 2008; Zhu et al., 2014; Jeene et al., 2019). Therefore, it is necessary to identify new therapeutic targets for PSCCE.

The human CD155 molecule was first discovered by Mendelsohn et al. (1989) and it has since been found to be broadly upregulated in malignant cells in solid tumors (Takai et al., 2008; Chandramohan et al., 2017). CD155 has a similar structure to Nectins and participates in intercellular adhesion; therefore, it is also known as Necl-5 (Yong et al., 2019). Li et al. found that CD155-depleted tumor cells displayed slower tumor growth and reduced metastases, demonstrating the importance of the tumor-intrinsic role of CD155 (Li et al., 2018). In addition, the presence of CD155 on the surface of cancer cells has been shown to promote tumor invasiveness, and its upregulation in tumor-infiltrating myeloid cells restrains antitumor immunity by impairing the function of antitumor T lymphocytes and NK cells (Bronte, 2018). Therefore, the role of CD155 in tumor proliferation and invasion in PSCCE is of considerable interest.

Similar to the inhibitory receptors, CTLA- 4 and PD-1, T cell immunoreceptor with Ig and ITIM domains (TIGIT) plays an important role in autoimmunity (Manieri et al., 2017) and has become the focus of much research in the immune checkpoint family due to its effect on T cell and NK cell exhaustion when bound to CD155 (Chan et al., 2014; Dougall et al., 2017; Zhang et al., 2018). Good therapeutic effects have been achieved in many preclinical models by blocking the interaction between CD155 and TIGIT (Blake et al., 2016; Dougall et al., 2017); however, its mechanism of action may depend on the cell type studied (Manieri et al., 2017). For instance, studies have confirmed that NK cells play important roles in the control of metastatic dissemination (López-Soto et al., 2017; Glasner et al., 2018). However, to our knowledge, no studies have yet focused on the CD155-TIGIT interaction or the relationship between CD155/TIGIT expression and the prognosis of PSCCE.

In this study, we evaluated CD155 and TIGIT expression using immunohistochemistry (IHC) and examined their prognostic effect on PSCCE. We found that high CD155 or TIGIT levels are associated with lower OS and progression-free survival (PFS). Therefore, this study is the first to assess the prognostic effects of CD155 and TIGIT expression in patients with PSCCE who have received CT or chemoradiotherapy (CRT).

\section{MATERIALS AND METHODS}

\section{Patients}

A total of 114 patients with PSCCE who consecutively underwent CT or CRT (including adjuvant CT/CRT) at our hospital between

\footnotetext{
Abbreviations: PSCCE, primary small cell carcinoma of the esophagus; CT, chemotherapy; RT, radiotherapy; CRT, chemoradiotherapy; TIGIT, T cell immunoreceptor with Ig and ITIM domains; IHC, immunohistochemistry; PFS, progression-free survival; OS, overall survival; PBS, phosphate-buffered saline; NSE, neuron-specific enolase; cyt-CD155, cytoplasmic CD155; m-CD155, membranous CD155; C-index, concordance index; DCA, decision curve analysis; LPS, lipopolysaccharides.
}

June 2006 and December 2018 were enrolled in this study according to the following eligibility criteria: (Lv et al., 2008) pathology-confirmed PSCCE, (Chen et al., 2016) Karnofsky performance status score $\geq 70$, (Wong et al., 2017) CT cycles $\geq 4$, and (Xu et al., 2017) RT dose $\geq 45$ Gy. The exclusion criteria were as follows: (Lv et al., 2008) history of other malignancies, (Chen et al., 2016) received RT only, and (Wong et al., 2017) incomplete follow-up data.

The clinicopathological data of the patients were collected from their medical records. Tumor stage was determined according to the TNM classification adopted by the International Union Against Cancer. This research project was approved by the Ethics Committee of Shandong Cancer Hospital and Institute and Shandong Provincial Hospital, and the requirement to obtain informed consent was waived due to the retrospective nature of the study.

\section{IHC Staining}

Samples obtained from pathological biopsy were routinely fixed in $10 \%$ neutral buffered formalin, embedded in paraffin, cut into $4-\mu \mathrm{m}$ sections, and dried for $1 \mathrm{~h}$ at $60^{\circ} \mathrm{C}$. The tissue slides were then deparaffinized in xylene, rehydrated using an alcohol gradient, and washed with purified water. Antigens were retrieved by heating the samples in citrate buffer $(\mathrm{pH}$ 6.0) for $20 \mathrm{~min}$ at $95^{\circ} \mathrm{C}$. After heating, the slides were allowed to cool to room temperature, briefly washed in phosphate-buffered saline (PBS), and endogenous peroxidase activity was neutralized using Peroxidase Block for $15 \mathrm{~min}$. The slides were then washed with PBS and treated with Protein Block for 15 min before being washed with PBS again and incubated with anti-CD155 (1:200 dilution, 81254S, CST, USA) and anti-TIGIT (1:200 dilution, NBP2-79793, Novus, USA) primary antibodies overnight at $4^{\circ} \mathrm{C}$. The negative controls were treated with PBS instead of the primary antibodies. On the 2 nd day, the slides were reheated at $37^{\circ} \mathrm{C}$ for $30 \mathrm{~min}$, washed with PBS, and treated with a Novolink polymer for $15 \mathrm{~min}$. A working solution consisting of 1:20 DAB chromogen in Novolink DAB substrate buffer was prepared and applied for $3 \mathrm{~min}$ and then the slides were counterstained with Novolink hematoxylin for $3 \mathrm{~min}$, dehydrated, and placed on cover slips. All images were recorded using an Olympus BX53 fluorescent microscope (Tokyo, Japan).

\section{Immunostaining Scoring}

CD155 expression was assessed semi-quantitatively according to its staining intensity in cancer cells, which was evaluated as follows: 0 (no staining), 1 (low staining), 2 (moderate staining), and 3 (high staining). No or low staining was defined as negative, and moderate or high staining was defined as positive. The number of CD155-positive PSCCE cells was determined

TABLE 1 | CD155 expression in PSCCE and adjacent tissues from 20 postoperative patients.

\begin{tabular}{lcccc}
\hline & CD155-positive & $\boldsymbol{p}$-value & TIGIT-positive & $\boldsymbol{p}$-value \\
\hline PSCCE & $12(60 \%)$ & 0.003 & $13(65 \%)$ & $<0.001$ \\
Matched adjacent tissues & $3(15 \%)$ & & $2(10 \%)$ &
\end{tabular}



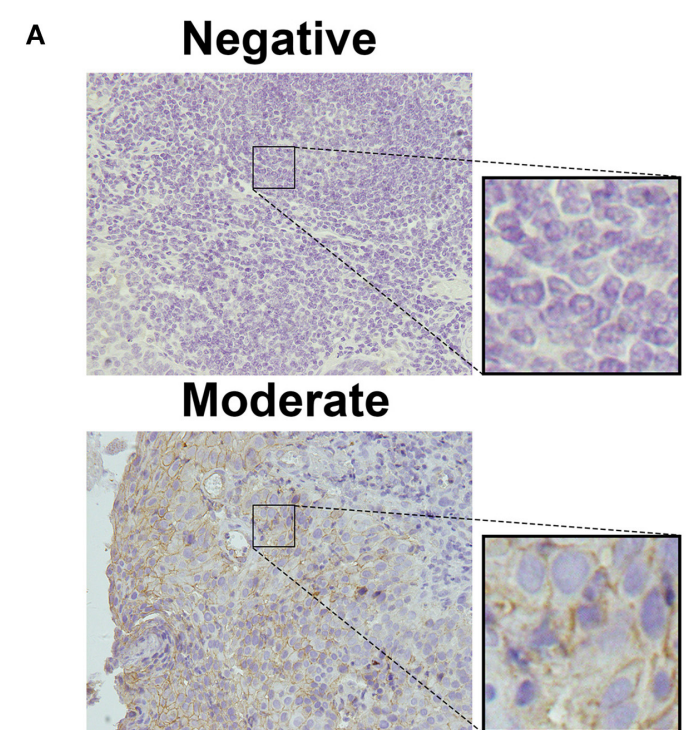

\section{Moderate}

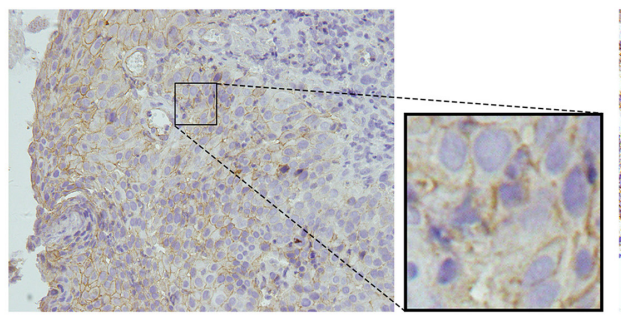

B

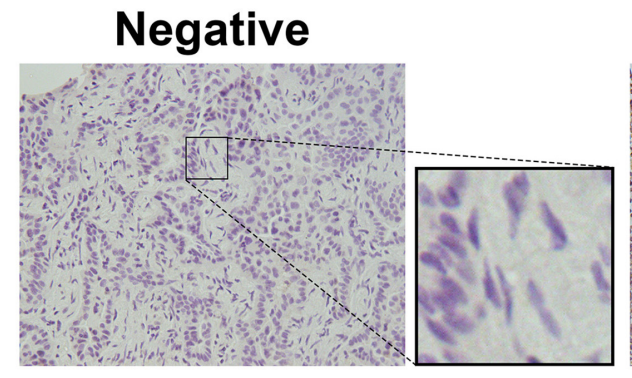

Low

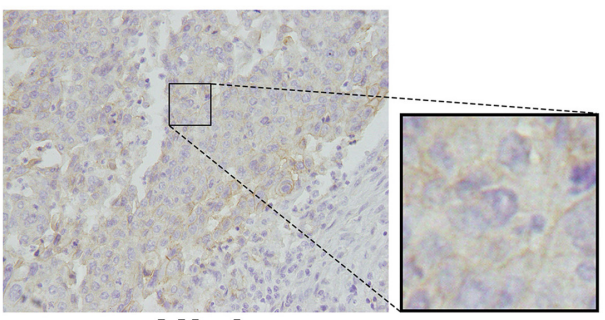

High

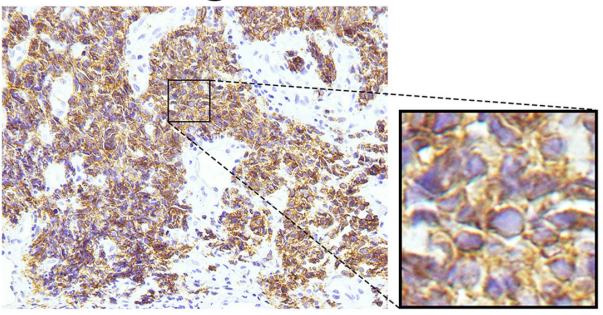

\section{Positive}

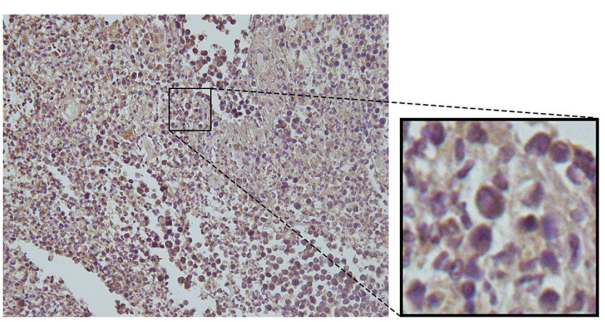

FIGURE 1 | CD155 and TIGIT expression characteristics in PSCCE tissues. Representative images for (A) negative, low, moderate, and high CD155 expression; (B) negative and positive TIGIT expression (original magnification $\times 400$ ).
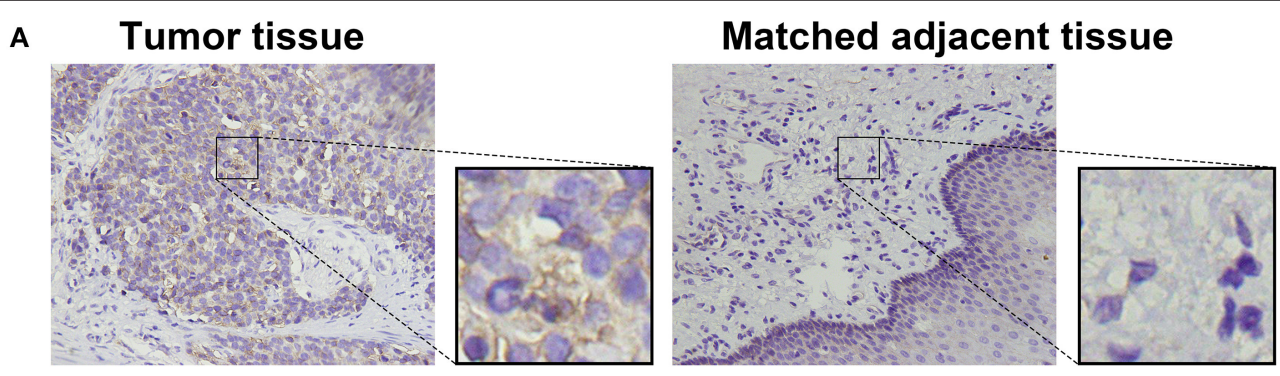

B
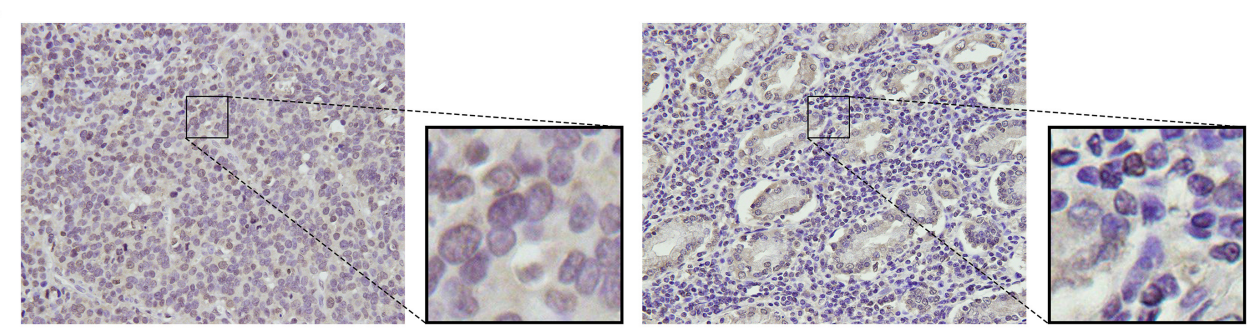

FIGURE 2 | Representative images from IHC analyses of (A). CD155 expression and (B). TIGIT expression in PSCCE tissue sections of different stages (original magnification $\times 400$ ). 


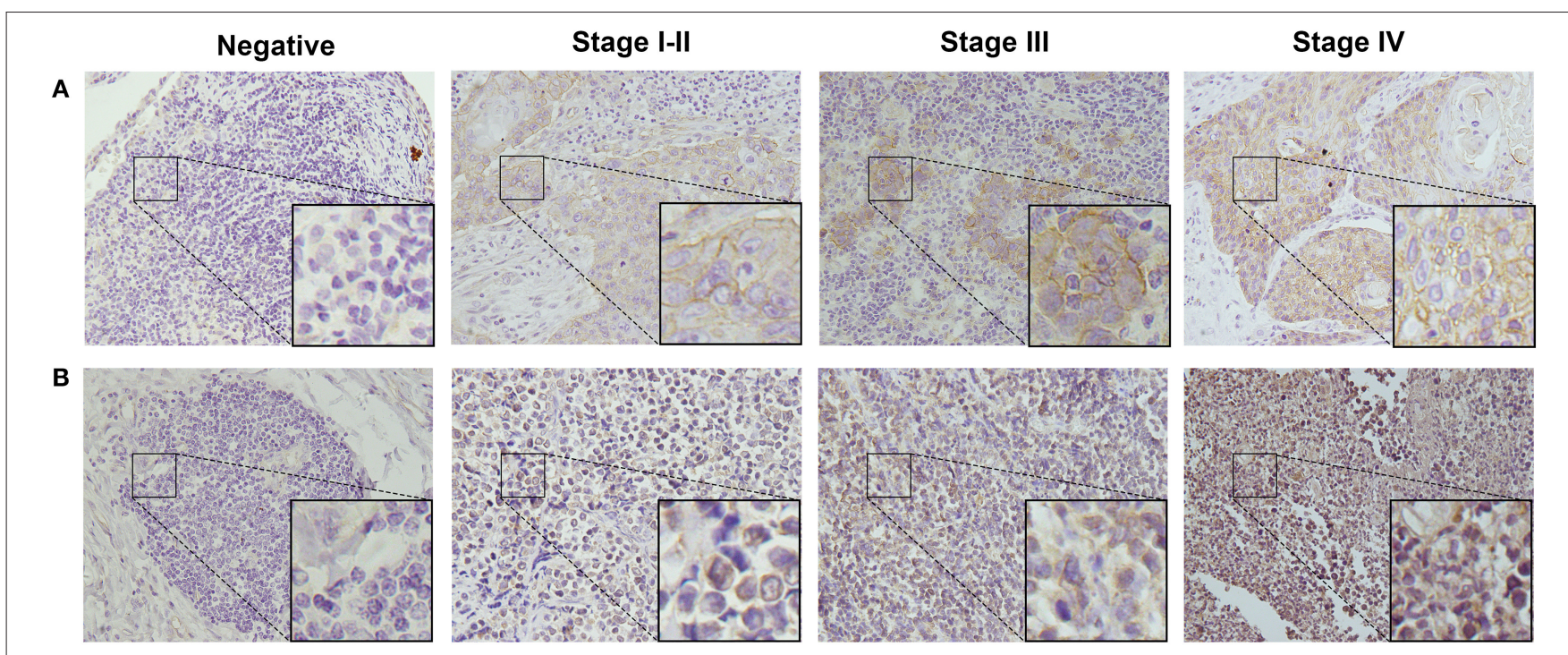

FIGURE 3 | Representative images comparing CD155 (A) and TIGIT (B) expression in tumor and matched adjacent tissues (original magnification $\times 400$ ).

quantitatively by averaging the number of positively stained cells in five randomly selected high-power fields at $400 \times$. The percentage of stained cells $(0-100 \%)$ was multiplied by the corresponding intensity score to obtain an intensity percentage score (0-300). TIGIT expression was assessed manually and semi-quantitatively in tumor cells as follows: $\leq 5 \%$ staining was considered negative and $>5 \%$ staining was scored as positive.

\section{Follow-Up}

Each patient was followed up every 3 months after treatment for the 1st year and every 6 months thereafter. Follow-up visits included physical examination, abdominal ultrasonography, chest computed tomography, and brain magnetic resonance imaging. Patients were excluded if they did not exhibit endevents at the end of the study. Follow-up was conducted until December 2019.

\section{Statistical Analysis}

Descriptive statistics for clinicopathological features were estimated using a simple frequency. Chi-square and Fisher's exact tests were used for categorical variables. Survival was estimated using the Kaplan-Meier method and log-rank test. Univariate and multivariate Cox regression hazards models were used to evaluate survival risk factors. Variables with a $p$-value of $<0.2$ from univariate analysis were subjected to multivariate analysis. The clinically significant variables calculated in multivariate analysis $(p<0.2)$ were integrated into a nomogram to predict the OS of PSCCE patients. Nomogram performance in terms of discrimination and calibration ability was evaluated using the concordance index (C-index) and Hosmer-Lemeshow-type $\chi^{2}$ statistics. The clinical utility of the nomogram was assessed via decision curve analysis (DCA). Statistical analyses were conducted using SPSS 22.0 (IBM, Armonk, NY, USA) and R software (version 3.5.3). $p$-values of $<0.05$ were considered statistically significant.

\section{RESULTS}

\section{Clinicopathological Characteristics of Patients}

The clinicopathological characteristics of the 114 patients with PSCCE included in this study are summarized in Table 1. Forty patients received CT and 74 received CRT. The age of the patients ranged from 41 to 78 years (median, 66 years). Eighty-four $(73.7 \%)$ of the patients were males, $61(53.5 \%)$ smoked, and $60(52.6 \%)$ had a history of drinking. Tumor size ranged from 2.0 to $14.0 \mathrm{~cm}$ (median 5). The distribution of TNM stages T1-4 in ascending order was $3,48,48$, and 15; N0-3 was 17, 51, 27, 19; M0-1 was 75 and 39; and TNM stages I-IV was 1, 21, 51, and 41 , respectively.

\section{CD155 and TIGIT Are Overexpressed in PSCCE Tissues}

To detect CD155 and TIGIT expression in the 114 cases of PSCCE, we performed IHC. CD155 was positively expressed in $67(58.8 \%)$ patients (Figure 1A), while TIGIT was positively expressed in $64(56.1 \%)$ patients (Figure 1B). The expression levels of CD155 and TIGIT increased with the disease progress (Figures 2A,B). In addition, we collected tumor and matched adjacent tissues from 20 patients with PSCCE who had undergone radical esophagectomy, finding that $\mathrm{CD} 155$ was positively expressed in $60.0 \%(12 / 20)$ of the tumor tissues compared to $15.0 \%$ of the matched adjacent tissues $(3 / 20)$. Similarly, the rate of TIGIT positivity was higher in tumor tissues than in the matched adjacent tissues (65 vs. $10 \%, p<$ 0.001), as shown in Table 1 and Figures 3A,B. These results imply that CD155 and TIGIT are overexpressed in tumor tissues of PSCCE. 
TABLE 2 | Cohort characteristics of 114 patients with PSCCE.

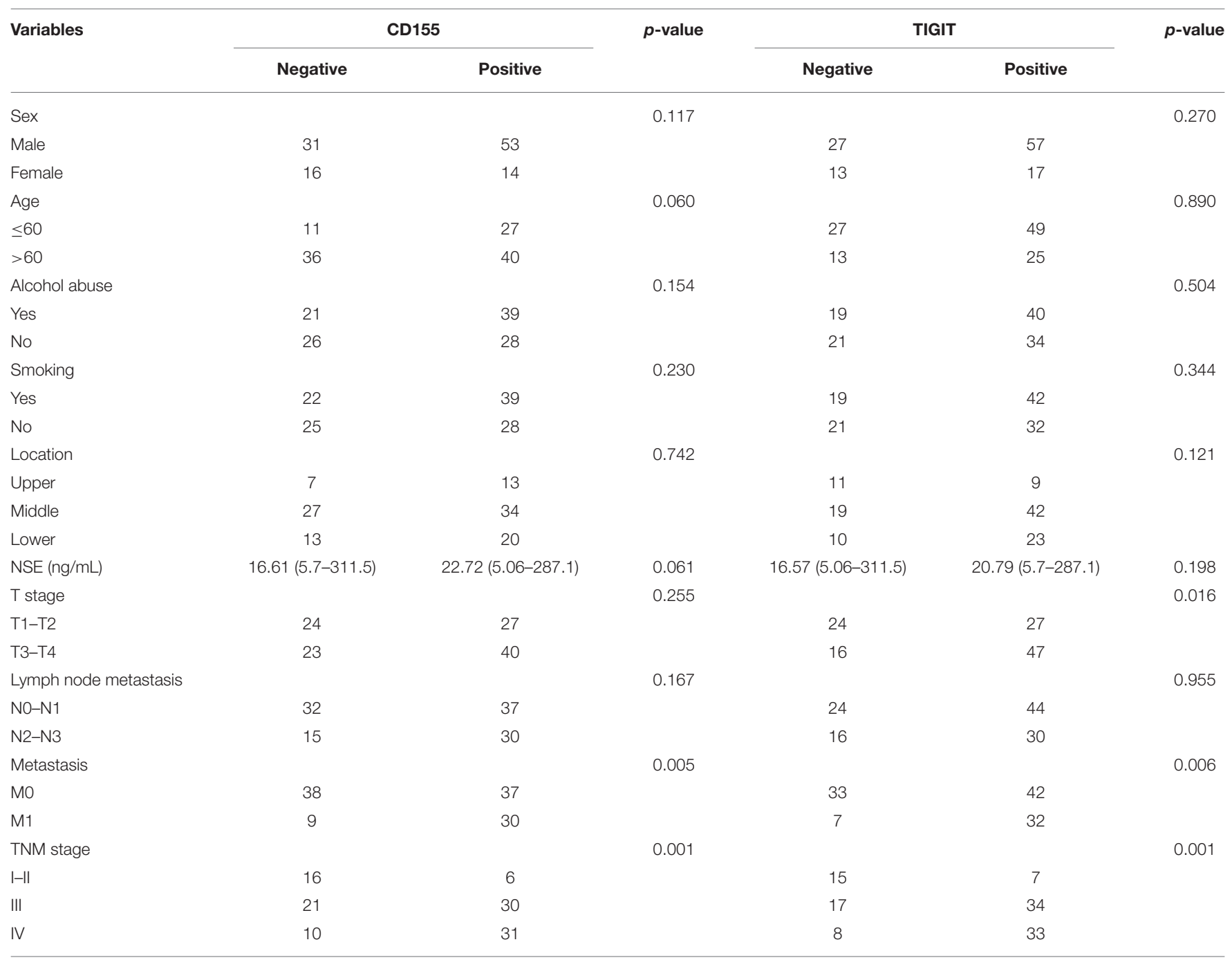

\section{CD155 and TIGIT Expression Correlates With the Clinicopathological Characteristics of PSCCE Patients}

The correlations between CD155 and TIGIT expression and the clinical parameters of patients with PSCCE are summarized in Table 2. CD155 expression did not correlate with sex, age, alcohol and smoking history, tumor location, $\mathrm{T}$ stage, or lymph node status; however, CD155 expression was higher in patients with larger tumors ( $p<0.001$, Figure 4A) and was significantly associated with TNM stage ( $p<0.001$, Figure 4B). Moreover, patients who developed lymphatic or distant metastases had significantly higher CD155 IHC scores than those with no evidence of metastasis ( $p<0.01$, Figures 4C,D), as did patients with higher Ki-67 scores ( $p=0.008$, Figure 4E). Conversely, there was no significant correlation between neuron-specific enolase (NSE) and CD155 expression ( $p=0.061$, Table 2). CD155 expression positively correlated with TIGIT $(p<0.001$, Figure 4F), and the proportion of TIGIT-positive patients tended to increase with advanced tumor size, T stage, distant metastasis, and TNM stage (Table 2, Figures 4G-J). Other covariates (age, sex, alcohol abuse, smoking, location, NSE level, and lymph node metastasis) were not notably associated with TIGIT expression. Together, these results suggest that CD155 and TIGIT are associated with tumor progression and tumor metastasis in PSCCE.

\section{Effect of CD155 and TIGIT Expression on OS and PFS in PSCCE}

The CD155-positive group had a shorter OS than the negative group (15 vs. 24 months, $p=0.001$, Figure 5A) as well as a lower PFS ( 9 vs. 15 months, $p=0.002$, Figure 5D). Similarly, TIGIT-positive patients had a shorter OS than did TIGITnegative patients ( 15 vs. 26 months, $p=0.001$, Figure 5B) and displayed a lower PFS ( 9 vs. 13 months, $p=0.034$, Figure 5E). We found that patients positive for both CD155 and TIGIT had the shortest OS (mean OS $=14$ months), whereas double negative expression was linked to the longest OS (mean OS = 27 months), while patients that were positive only for CD155 

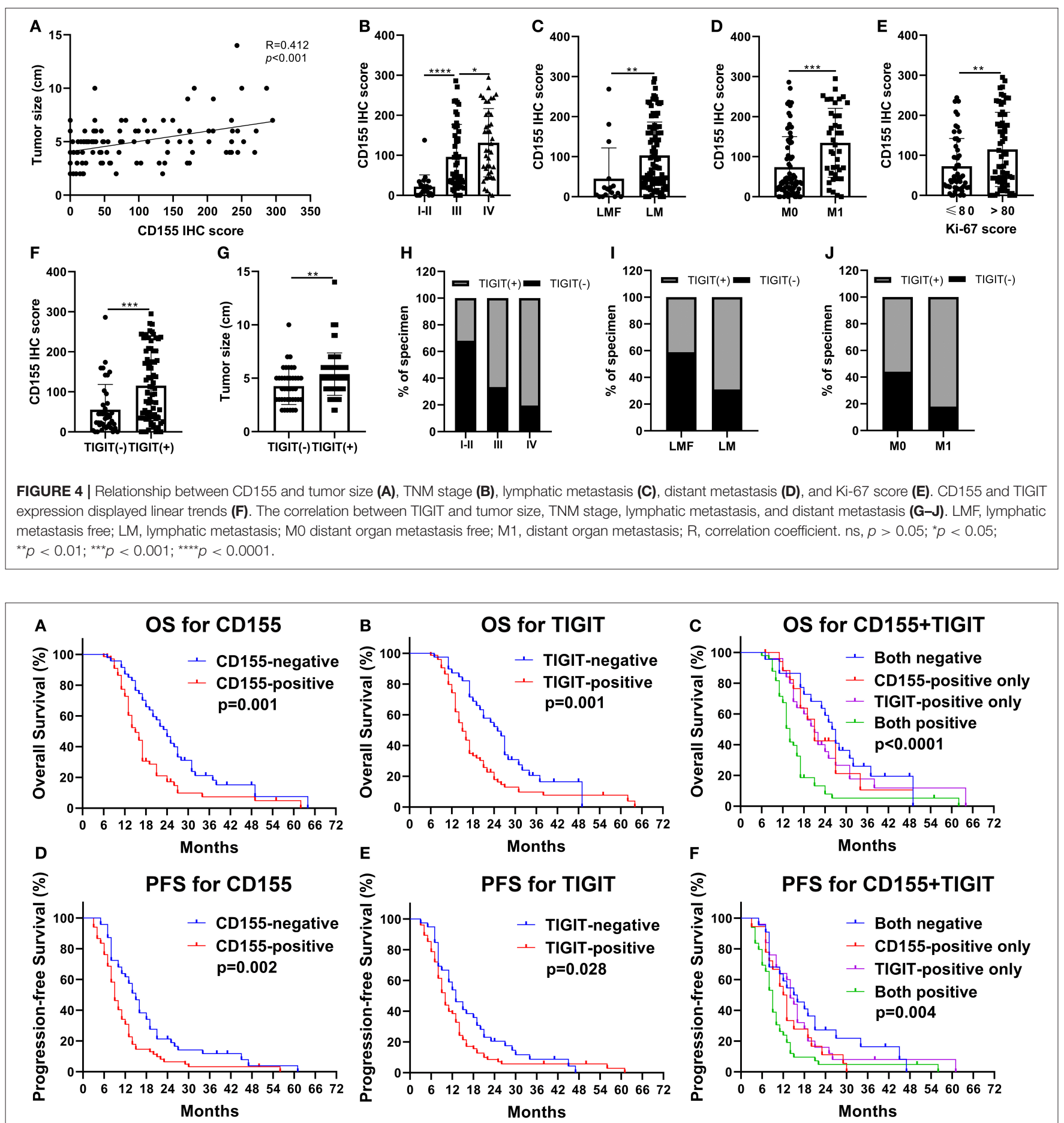

FIGURE 5 | Kaplan-Meier curves of OS for CD155 (A) and TIGIT (B) expression and their combination (C). Kaplan-Meier curves of PFS for CD155 (D) and TIGIT (E) expression and their combination (F).

or TIGIT positive had the same OS (mean OS $=21$ months) $(p<0.001$, Figure 5C). In addition, patients positive for both CD155 and TIGIT had a shorter mean PFS than those negative for both ( 9 vs. 17 months), while patients that were positive only for CD155 or TIGIT positive had the similar PFS(13 vs. 14 months) ( $p=0.004$, Figure 5F). These findings suggested that analysis of CD155 and TIGIT status might predict the prognosis of PSCCE.

Risk Factors for Poor Prognosis in PSCCE

Next, we analyzed possible prognostic factors in patients with PSCCE using univariate and multivariate Cox regression 
TABLE 3 | Univariate and multivariate analyses of prognostic markers for OS in PSCCE.

\begin{tabular}{|c|c|c|c|c|c|c|}
\hline \multirow[t]{2}{*}{ Variable } & \multicolumn{3}{|c|}{ Univariate analysis } & \multicolumn{3}{|c|}{ Multivariate analysis } \\
\hline & HR & $95 \% \mathrm{Cl}$ & $p$-value & HR & $95 \% \mathrm{Cl}$ & $p$-value \\
\hline \multicolumn{7}{|l|}{ CD155 expression } \\
\hline $\begin{array}{l}\text { High vs. low or } \\
\text { negative }\end{array}$ & 1.966 & $1.292-2.990$ & 0.002 & 1.646 & $1.006-2.691$ & 0.047 \\
\hline \multicolumn{7}{|l|}{ TIGIT expression } \\
\hline $\begin{array}{l}\text { Negative vs. } \\
\text { positive }\end{array}$ & 2.012 & $1.300-3.116$ & 0.002 & 1.590 & $0.970-2.607$ & 0.066 \\
\hline Sex & & & & & & \\
\hline male vs. female & 1.192 & $0.758-1.874$ & 0.447 & & & \\
\hline \multicolumn{7}{|l|}{ Age (years) } \\
\hline$\leq 60$ vs. $>60$ & 0.971 & $0.635-1.484$ & 0.892 & & & \\
\hline \multicolumn{7}{|l|}{ Alcohol abuse } \\
\hline Yes vs. no & 1.200 & $0.803-1.792$ & 0.373 & & & \\
\hline \multicolumn{7}{|l|}{ Smoking } \\
\hline Yes vs. no & 1.128 & $0.754-1.688$ & 0.559 & & & \\
\hline \multicolumn{7}{|l|}{ Location } \\
\hline $\begin{array}{l}\text { Upper vs. middle } \\
\text { vs. lower }\end{array}$ & 1.099 & $0.818-1.478$ & 0.530 & & & \\
\hline \multicolumn{7}{|l|}{ Length (cm) } \\
\hline $\begin{array}{l}\leq 4 \text { vs. }>4 \\
\text { T stage }\end{array}$ & 1.421 & $0.941-2.147$ & 0.095 & 1.143 & $0.711-1.836$ & 0.581 \\
\hline T1-2 vs. T3-4 & 1.742 & $1.145-2.651$ & 0.010 & 1.125 & $0.709-1.784$ & 0.618 \\
\hline \multicolumn{7}{|l|}{$\begin{array}{l}\text { Lymph node } \\
\text { metastasis }\end{array}$} \\
\hline N0-1 vs. N2-3 & 1.891 & $1.256-2.846$ & 0.002 & 1.001 & $0.591-1.698$ & 0.996 \\
\hline \multicolumn{7}{|l|}{ Distant metastasis } \\
\hline M0 vs. M1 & 2.318 & $1.564-3.436$ & $<0.001$ & 0.676 & $0.281-1.626$ & 0.382 \\
\hline \multicolumn{7}{|l|}{ TNM stage } \\
\hline $\begin{array}{l}\text { I-II vs. III vs. IV } \\
\text { Therapeutic } \\
\text { strategy }\end{array}$ & 2.030 & $1.521-2.710$ & $<0.001$ & 1.834 & $0.954-3.523$ & 0.069 \\
\hline CT vs. CRT & 0.477 & $0.313-0.728$ & 0.001 & 0.502 & $0.309-0.814$ & 0.005 \\
\hline NSE & & & & & & \\
\hline $\begin{array}{l}>18.26 \text { vs. } \\
\leq 18.26\end{array}$ & 1.479 & $0.971-2.253$ & 0.068 & 1.002 & $0.999-1.005$ & 0.239 \\
\hline Ki67 (\%) & & & & & & \\
\hline$\geq 80$ vs. $<80$ & 1.297 & $0.859-1.958$ & 0.216 & & & \\
\hline
\end{tabular}

HR, Hazard ratio; Cl, confidence interval.

(Table 3). Univariate analysis showed that CD155 and TIGIT expression, $\mathrm{T}$ stage, lymph node and distant metastasis, TNM stage, and therapeutic strategy were all associated with OS, whereas sex, age, alcohol and smoking history, tumor location, tumor size, and Ki-67 score were not. Subsequent multivariate Cox regression analysis revealed that CD155 expression and therapeutic strategy were independent prognostic factors for OS.

\section{Construction and Validation of the Nomogram}

The predictive ability of the model was assessed by calculating the C-index, which was 0.724 (95\%CI: 0.695-0.753), demonstrating good predictive accuracy for the nomogram. The nomogram predicting the OS of patients with PSCCE is displayed in Figure 6A. Each factor was ascribed a weighted point total that indicated a survival prognosis. 1 and 3-year survival probabilities were measured using this nomogram. The calibration curve showed favorable overlap with the reference line, demonstrating the good performance of the nomogram (Figure 6B). The DCA exhibited satisfactory benefits of the nomogram at the threshold probabilities (Figure 6C).

\section{Effect of Combined CD155 and TIGIT Expression on Clinical Outcome in Different PSCCE Subsets}

We found that OS rates decreased dramatically with successive increases in TNM staging (Figure 7A, $p<0.001$ ), indicating the value of TNM stage for predicting the prognosis of PSCCE. Moreover, combined CD155 and TIGIT expression was a statistically significant prognostic indicator for OS in patients with stage III-IV, T stage, N0-1 stage, and M0 stage PSCCE (Figures 7B-I, $p<0.05$ ). Taken together, these findings suggest that CD155 and TIGIT expression can be used as an independent prognostic indicator for patients with PSCCE.

\section{DISCUSSION}

CD155 is a member of the nectin-like family of adhesion molecules that has a wide variety of functions relevant to cancer, including tumor cell-intrinsic activities that regulate proliferation, adhesion, and migration as well as the ability to affect immune responses by binding to the immunomodulatory receptors DNAM-1, CD96, and TIGIT (Li et al., 2018). The expression of CD155 in tumor tissues and stroma has been widely recognized in recent years. Recent studies have found that CD155 is expressed at low levels in many normal cells, such as epithelial cells, endothelial cells, nerve cells, and fibroblasts (Yong et al., 2019), but is expressed at high levels in many tumors, such as colorectal cancer (Masson et al., 2001), gastric cancer (He et al., 2017), ovarian cancer (Carlsten et al., 2009), melanoma (Inozume et al., 2016), lung cancer (Sun et al., 2020), and breast cancer (Triki et al., 2019). In this study, we found that CD155 expression was higher in PSCCE than in matched adjacent tissues $(p=0.003)$ and that the rate of positive CD155 expression was slightly higher than that reported in previous studies (He et al., 2017; Yong et al., 2019; Sun et al., 2020). This may be related to the fact that PSCCE is a highly malignant and aggressive tumor. Importantly, our findings are similar to those reported for other tumor tissue types and suggest that CD155 expression may also play an important role in the development and treatment response of PSCCE.

We found that CD155 expression was related to tumor size, Ki-67, lymph node metastasis, distant metastasis, and TNM stage, suggesting that CD155 overexpression could be associated with tumor metastasis and proliferation. Previously, Li et al. (2018) showed that CD155 expression in tumor and hematopoietic cells contributed toward tumor progression via non-redundant mechanisms. Meanwhile, Sloan et al. (2005) 


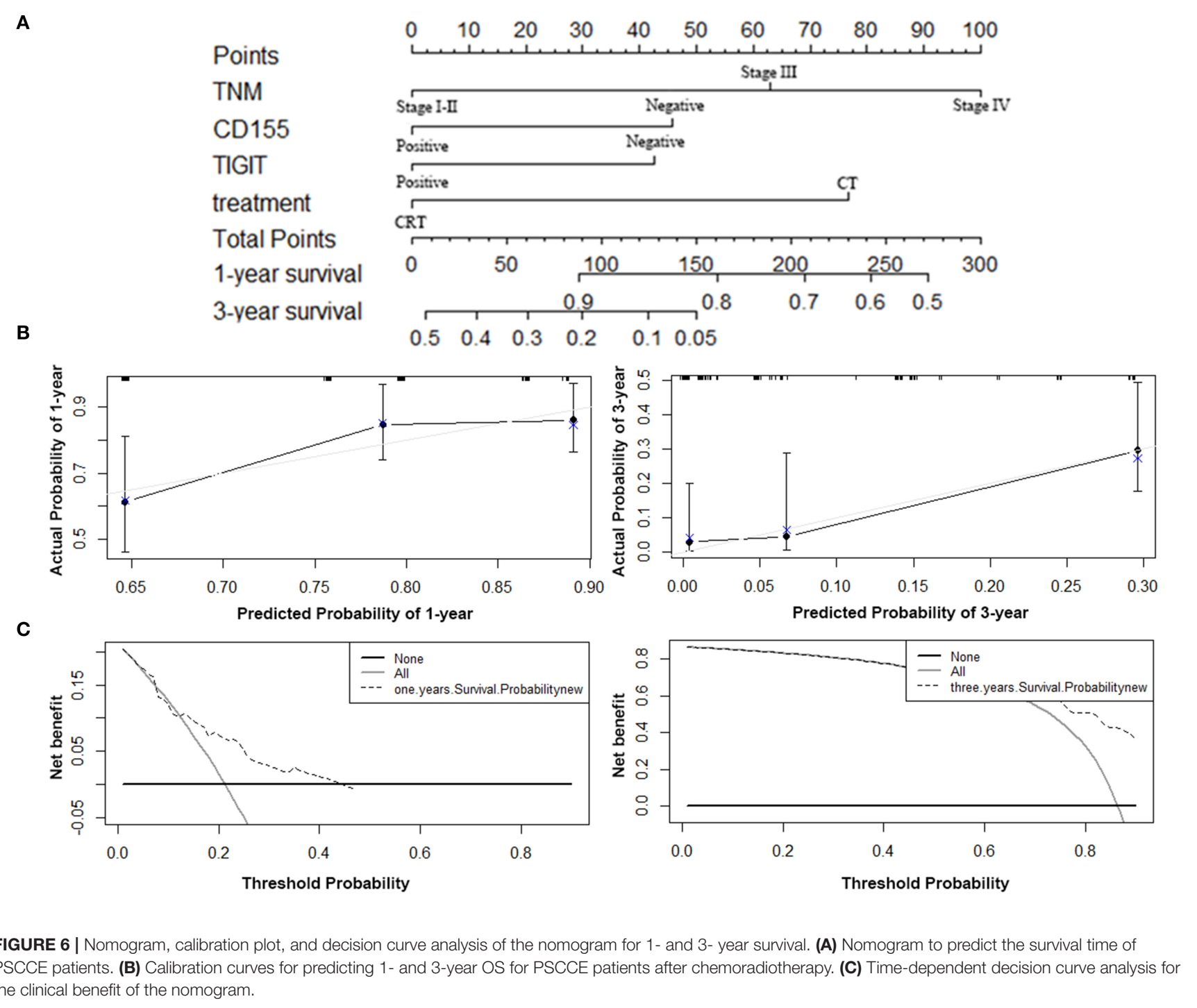

found that CD155 expression reduced substrate adhesion, cell spreading, focal adhesion density, and the number of actin stress fibers in a substrate-dependent manner. The depletion of endogenous CD155 in human glioma cells inhibited their migration, increased cell spreading, and downregulated the same signaling pathway. Tane et al. (2013) found that CD155 knockdown by RNAi transfection in bronchioloalveolar carcinoma cells slowed growth and migration and decreased their invasive ability. PSCCE is a highly invasive tumor that often displays high $\mathrm{Ki}-67$ expression in later stages. In this study, we found that CD155 expression was consistent with Ki-67 expression and tumor stage, partially confirming its role in tumor proliferation. Li et al. (2018) demonstrated a previously unrecognized immunosuppressive role for hostderived CD155 that is independent of tumor-derived CD155, which promotes tumor growth and metastasis via tumor-intrinsic mechanisms related to cell migration and survival. The loss of both host- and tumor-derived CD155 led to the greatest reduction in tumor growth and improved responses to anti-PD1 or combined anti-PD-1 and anti-CTLA4 blockade. Triki et al. (2019) also reported that tumor cytoplasmic CD155 (cyt-CD155) was associated with lymphovascular invasion, while membranous CD155 (m-CD155) correlated strongly with the presence of tumor-infiltrating NK cells. CD155 may therefore play an important role in PSCCE proliferation and metastasis; however, the molecular mechanisms require further investigation.

TIGIT is an inhibitory checkpoint receptor that has become the focus of much research due to its role in $\mathrm{T}$ cell and $\mathrm{NK}$ cell exhaustion when bound to CD155 (Dougall et al., 2017; O'Donnell et al., 2019). Notably, TIGIT is highly expressed in CD8+T cells, CD4+T cells, and NK cells during tumor infiltration (Song et al., 2018; Josefsson et al., 2019; Lupo and Matosevic, 2020). In this study, we found that TIGIT was highly expressed in tumor tissues and that high TIGIT expression predicted a more advanced disease stage and worse prognosis in PSCCE. TIGIT has recently emerged as a major 


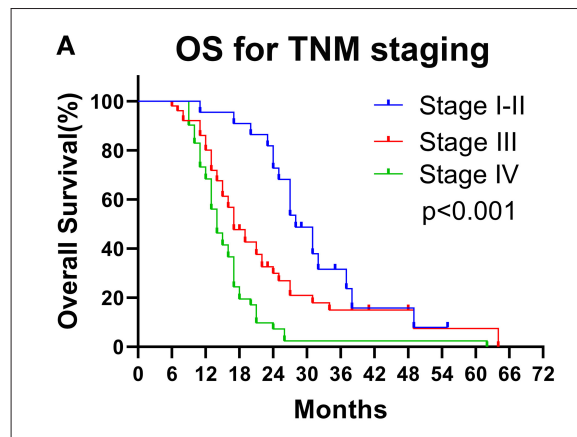

D T1-2 OS for CD155+TIGIT

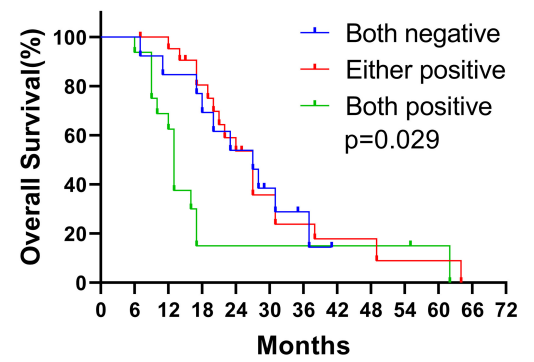

G N2-3 OS for CD155+TIGIT

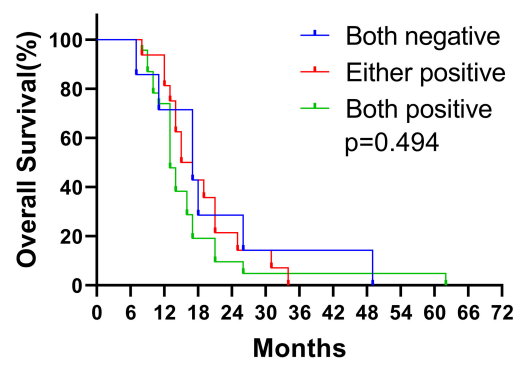

B Stage I-II OS for CD155+TIGIT C

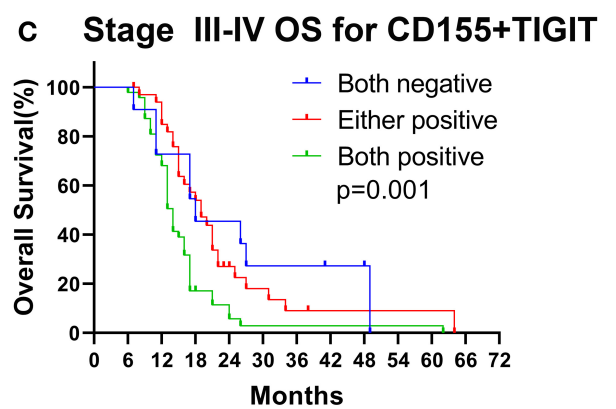

E

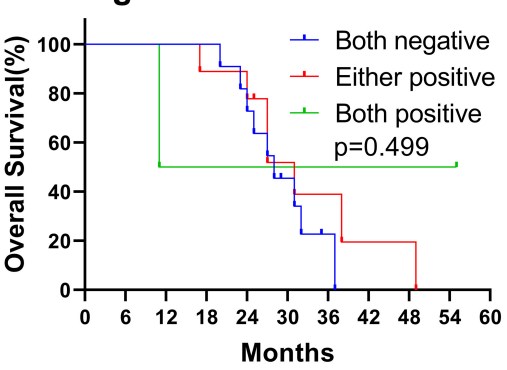

F N0-1 OS for CD155+TIGIT
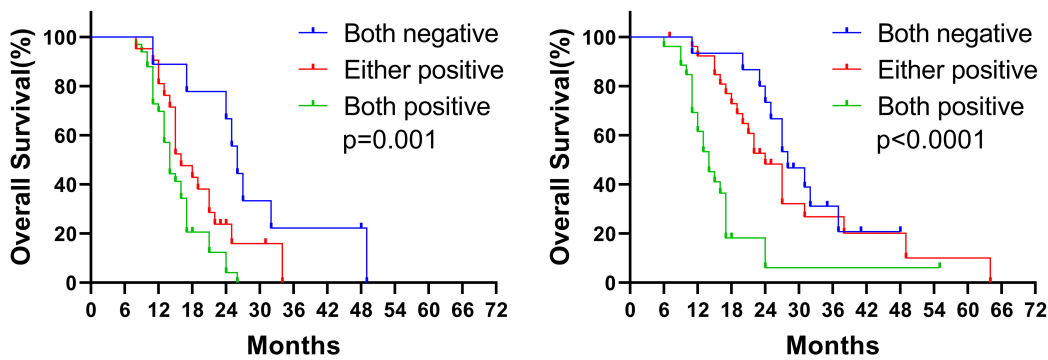

H M0 OS for CD155+TIGIT
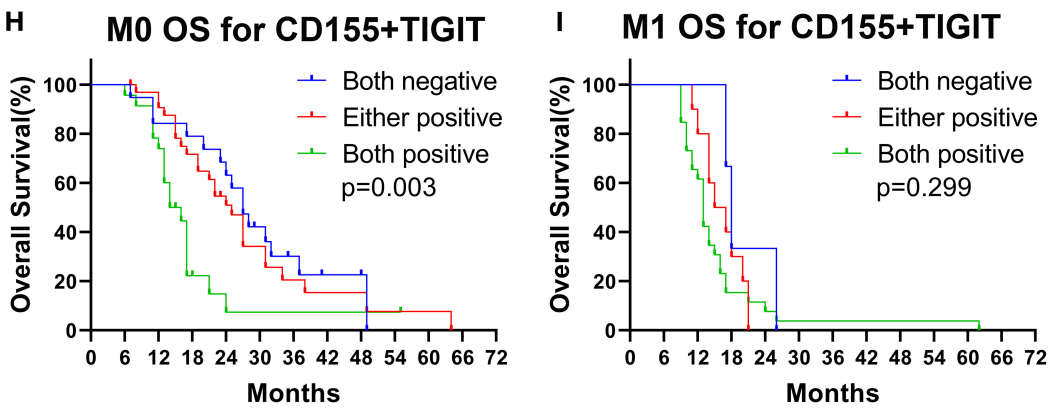

FIGURE 7 | Subgroup OS analysis according to (A-C) TNM staging, (D,E) T staging, (F,G) N staging, (H,I) M staging.

target in cancer immunotherapy (Harjunpää and Guillerey, 2020). Indeed, $\mathrm{Wu}$ et al. found that CD155/TIGIT signaling blockade reverses $\mathrm{T}$ cell exhaustion and enhances antitumor capabilities in head and neck squamous cell carcinoma (Wu et al., 2019). Targeting CD155/TIGIT enhanced the CD8+T cell reaction, while the combined targeting of TIGIT and PD1 further enhanced CD8+T cell activation (He et al., 2017). NK cells are an important component of innate immunity that are thought to play an important role in the early stages of cancer elimination and preventing metastasis (Manieri et al., 2017; Jewett et al., 2018). High TIGIT expression has been shown to predict an increased risk of metastasis and thus may be a reason for early PSCCE metastasis. In addition, TIGIT blockade was shown to prevent NK cell exhaustion and promote NK cell-dependent tumor immunity in several tumorbearing mouse models (Zhang et al., 2018). Many conventional therapeutic strategies, including $\mathrm{CT}$ and $\mathrm{RT}$, remain fairly unsuccessful when treating poorly differentiated tumors (Jewett et al., 2018); however, the application of immunotherapy in small cell lung carcinoma has achieved encouraging results in recent years (Horn et al., 2018; Nishio et al., 2019). Therefore, blocking the interaction between CD155 and TIGIT may be a good addition to conventional treatment strategies for PSCCE.

In this study, we found that CD155 expression was an independent prognostic factor for OS. Moreover, we found that the combined prognostic value of CD155 and TIGIT was statistically significant for subgroups including stage III-IV, T stage, N0-1 and M0 stage, further emphasizing the utility of CD155 and TIGIT for predicting the prognosis of PSCCE after CT/CRT. The nomogram including CD155 and TIGIT demonstrated good performance in clinical utility. Previously, Yong et al. (2019) found that breast cancer patients with high CD155 expression had poor OS rates, while Sun et al. (2020) showed that CD155 expression was an independent risk factor for lung adenocarcinoma and that patients with high CD155 expression presented shorter OS and PFS. However, $\mathrm{Qu}$ et al. found that positive CD155 expression was associated 
with a good prognosis (Qu et al., 2015). Triki et al. (2019) revealed that patients with high cyt-CD155 levels had a significantly worse OS and death-free survival than those with low expression, whereas high m-CD155 levels correlated with a better prognosis. Although our conclusion differs from these previous reports, it also suggests that cyt-CD155 may play a series of roles in the cytoplasm that enhance cell proliferation and invasion ability, whereas m-CD155 affects the tumor microenvironment via intercellular interactions. Therefore, how each CD155 form plays a role in tumor progression requires further study.

In addition, the understanding of the mechanisms responsible for CD155 and TIGIT overexpression may allow the development of new treatment strategies. Zhang et al. found that FOXP3 directly regulates TIGIT expression in CD4+CD25+ Treg cells because there is an overlapping region of differential methylation and FOXP3 binding peak in the TIGIT promoter (Zhang et al., 2013). Recent research has found that TIGIT upregulation can be inhibited by Tox- deletion in tumor-specific T cells (Scott et al., 2019). Other previous studies have found that TIGIT expression was upregulated in aged $\mathrm{T}$ cell populations and increased in CD8+ $\mathrm{T}$ cells by $3 \times 8 \mathrm{~Gy}$ RT but decreased by $18 \mathrm{x} 2 \mathrm{~Gy}$; however, the mechanisms are unclear (Song et al., 2018; Grapin et al., 2019). Furthermore, it has been revealed that chemotherapeutic agents, reactive oxygen species and reactive nitrogen species can upregulate CD155 expression by damaging the DNA of tumor cells (Gao et al., 2017). Moreover, CD155 expression on dendritic cells can be upregulated by lipopolysaccharides (LPS) (Gilfillan et al., 2008), and exposure of mouse bone marrow-derived macrophages and B cells to LPS could increase CD155 level in an NF- $\kappa$ B dependent manner (Escalante et al., 2011; Kamran et al., 2013). However, the mechanisms responsible for the upregulation of CD155 and TIGTI are not fully understood and further studies are needed.

Despite our important findings, this study has some limitations. Firstly, although CD155 is also expressed in myeloid cells, we only evaluated its expression in tumor cells. Secondly, we did not evaluate the expression of CD155 ligands (CD96/ CD226), which also influence its interaction with immune cells; however, these issues will be addressed in the follow-up study.

In conclusion, our results demonstrate that CD155 and TIGIT are abnormally overexpressed in PSCCE cells and that higher levels of CD155 are related to a worse prognosis in patients with PSCCE. Therefore, CD155 expression may be used as an independent prognostic indicator for patients with PSCCE.

\section{REFERENCES}

Blake, S. J., Stannard, K., Liu, J., Allen, S., Yong, M. C., Mittal, D., et al. (2016). Suppression of metastases using a new lymphocyte checkpoint target for cancer immunotherapy. Cancer Discov. 6, 446-459. doi: 10.1158/2159-8290.CD-15-0944

Bronte, V. (2018). The expanding constellation of immune checkpoints: a DNAMic control by CD155. J. Clin. Invest. 128, 2199-2201. doi: 10.1172/JCI121229

Carlsten, M., Norell, H., Bryceson, Y. T., Poschke, I., Schedvins, K., Ljunggren, H. G., et al. (2009). Primary human tumor cells expressing CD155 impair
Our study improves our understanding of PSCCE checkpoint examination and provides a basis for further research on immunotherapy for PSCCE.

\section{DATA AVAILABILITY STATEMENT}

The original contributions presented in the study are included in the article/supplementary materials, further inquiries can be directed to the corresponding authors.

\section{ETHICS STATEMENT}

The studies involving human participants were reviewed and approved by This research project was approved by the Ethics Committee of Shandong Cancer Hospital and Institute and Shandong Provincial Hospital Affiliated to Shandong First Medical University, and Shandong Provincial Hospital. Written informed consent for participation was not required for this study in accordance with the national legislation and the institutional requirements.

\section{AUTHOR CONTRIBUTIONS}

KZ: data collection, statistics, and original draft. LM, LF, and $\mathrm{ZH}$ : data collection, formal analysis, and resources. XM: conceptualization, review, and editing the manuscript. JY: monitored the clinical trial. All authors contributed to the article and approved the submitted version.

\section{FUNDING}

This work was supported by the Innovation Project of Shandong Academy of Medical Sciences (grant number 2019-04), Academic Promotion Program of Shandong First Medical University (grant number 2019ZL002), National Natural Science Foundation of China (grant numbers 81972796), Natural Science Foundation of Shandong Province (grant numbers ZR2019MH010), and Yantai Science and Technology Innovation Development Program (grant number 2020YT06121070).

\section{ACKNOWLEDGMENTS}

We appreciate the technical support of Ran Wei, Xia Li, Xiaoyang Yin, and Liangchao Sun. We would like to thank Editage (www.editage.cn) for English language editing.

tumor targeting by down-regulating DNAM-1 on NK cells. J. Immunol. 183, 4921-4930. doi: 10.4049/jimmunol.0901226

Chan, C. J., Martinet, L., Gilfillan, S. F., Souza-Fonseca-Guimaraes, Chow, M. T., Town, L., et al. (2014). The receptors CD96 and CD226 oppose each other in the regulation of natural killer cell functions. Nat. Immunol. 15, 431-438. doi: $10.1038 /$ ni. 2850

Chandramohan, V., Bryant, J. D., Piao, H., Keir, S. T., Lipp, E. S., Lefaivre, M., et al. (2017). Validation of an immunohistochemistry assay for detection of CD155, the poliovirus receptor, in malignant gliomas. Arch. Pathol. Lab. Med. 141, 1697-1704. doi: 10.5858/arpa.2016-0580-OA 
Chen, W., Zheng, R., Baade, P. D., Zhang, S., Zeng, H., Bray, F., et al. (2016). Cancer statistics in China, 2015. CA Cancer J. Clin. 66, 115-132. doi: $10.3322 /$ caac. 21338

Dougall, W. C., Kurtulus, S., Smyth, M. J., and Anderson, C. A. (2017). TIGIT and CD96: new checkpoint receptor targets for cancer immunotherapy. Immunol. Rev. 276, 112-120. doi: 10.1111/imr.12518

Escalante, N. K., von Rossum, A., Lee, M., and Choy, C. J. (2011). CD155 on human vascular endothelial cells attenuates the acquisition of effector functions in CD8 T cells. Arterioscler. Thromb. Vasc. Biol. 31, 1177-1184. doi: 10.1161/ATVBAHA.111.224162

Gao, J., Zheng, Q., Xin, N., Wang, W., and Zhao, C. (2017). CD155, an onco-immunologic molecule in human tumors. Cancer Sci. 108, 1934-1938. doi: $10.1111 /$ cas. 13324

Gilfillan, S., Chan, C. J., Cella, M., Haynes, N. M., Rapaport, A. S., Boles, K. S., et al. (2008). DNAM-1 promotes activation of cytotoxic lymphocytes by nonprofessional antigen-presenting cells and tumors. J. Exp. Med. 205, 2965-2973. doi: 10.1084/jem.20081752

Glasner, A., Levi, A., Enk, J., Isaacson, B., Viukov, S., Orlanski, S., et al. (2018). NKp46 receptor-mediated interferon- $\gamma$ production by natural killer cells increases fibronectin 1 to alter tumor architecture and control metastasis. Immunity 48, 107-119. doi: 10.1016/j.immuni.2017.12.007

Grapin, M., Richard, C., Limagne, E., Boidot, R., Morgand, V., Bertaut, A., et al. (2019). Optimized fractionated radiotherapy with anti-PD-L1 and anti-TIGIT: a promising new combination. J. Immunother. Cancer 7:160. doi: 10.1186/s40425-019-0634-9

Harjunpää, H., and Guillerey, C. (2020). TIGIT as an emerging immune checkpoint. Clin. Exp. Immunol. 200, 108-119. doi: 10.1111/cei.13407

He, W., Zhang, H., Han, F., Chen, X., Lin, R., Wang, W., et al. (2017). CD155T/TIGIT signaling regulates CD8(+) T-cell metabolism and promotes tumor progression in human gastric cancer. Cancer Res. 77, 6375-6388. doi: 10.1158/0008-5472.CAN-17-0381

Horn, L., Mansfield, A. S., A., Szczesna, Havel, L., Krzakowski, M., Hochmair, M. J., et al. (2018). First-line atezolizumab plus chemotherapy in extensive-stage small-cell lung cancer. N. Engl. J. Med. 379, 2220-2229. doi: 10.1056/NEJMoa1809064

Inozume, T., Yaguchi, T., Furuta, J., Harada, K., Kawakami, Y., and Shimada, S. (2016). Melanoma cells control antimelanoma CTL responses via interaction between TIGIT and CD155 in the effector phase. J. Invest. Dermatol. 136, 255-263. doi: 10.1038/JID.2015.404

Jeene, P. M., Geijsen, E. D., Muijs, C. T., Rozema, T., Aleman, B. M. P., Muller, K., et al. (2019). A nationwide analysis of treatment and outcome at patient level in locoregional disease. Am. J. Clin. Oncol. 42, 534-538. doi: 10.1097/COC.0000000000000546

Jewett, A., Kos, J., Fong, Y., Ko, M. W., Safaei, T., M., Nanut, M. P., et al. (2018). NK cells shape pancreatic and oral tumor microenvironments; role in inhibition of tumor growth and metastasis. Semin. Cancer Biol. 53, 178-188. doi: 10.1016/j.semcancer.2018.08.001

Josefsson, S. E., Beiske, K., Blaker, Y. N., Førsund, M. S., Holte, H., Østenstad, B., et al. (2019). TIGIT and PD-1 mark intratumoral T cells with reduced effector function in B-cell non-hodgkin lymphoma. Cancer Immunol. Res. 7, 355-362. doi: 10.1158/2326-6066.CIR-18-0351

Kamran, N., Takai, Y., Miyoshi, J., Biswas, S. K., Wong, J. S., and Gasser, S. (2013). Toll-like receptor ligands induce expression of the costimulatory molecule CD155 on antigen-presenting cells. PLoS ONE 8:e54406. doi: 10.1371/journal.pone.0054406

Li, X. Y., Das, I., Lepletier, A., Addala, V., Bald, T., Stannard, K., et al. (2018). CD155 loss enhances tumor suppression via combined host and tumorintrinsic mechanisms. J. Clin. Invest. 128, 2613-2625. doi: 10.1172/JCI98769

López-Soto, A., Gonzalez, S., Smyth, M. J., and Galluzzi, L. (2017). Control of metastasis by NK cells. Cancer Cell 32, 135-154. doi: 10.1016/j.ccell.2017.06.009

Lupo, K. B., and Matosevic, S. (2020). CD155 immunoregulation as a target for natural killer cell immunotherapy in glioblastoma. J. Hematol. Oncol. 13:76. doi: 10.1186/s13045-020-00913-2

Lv, J., Liang, J., Wang, J., Wang, L., He, J., Xiao, Z., et al. (2008). Primary small cell carcinoma of the esophagus. J. Thorac. Oncol. 3, 1460-1465. doi: 10.1097/JTO.0b013e31818e1247
Manieri, N. A., Chiang, E. Y., and Grogan, L. J. (2017). TIGIT: a key inhibitor of the cancer immunity cycle. Trends Immunol. 38, 20-28. doi: 10.1016/j.it.2016.10.002

Masson, D., Jarry, A., Baury, B., Blanchardie, P., Laboisse, C., Lustenberger, P., et al. (2001). Overexpression of the CD155 gene in human colorectal carcinoma. Gut 49, 236-240. doi: 10.1136/gut.49.2.236

Mendelsohn, C. L., Wimmer, E., and Racaniello, R. V. (1989). Cellular receptor for poliovirus: molecular cloning, nucleotide sequence, and expression of a new member of the immunoglobulin superfamily. Cell 56, 855-865. doi: 10.1016/0092-8674(89)90690-9

Meng, M. B., Zaorsky, N. G., Jiang, C., Tian, L. J., Wang, H. H., Liu, C. L., et al. (2013). Radiotherapy and chemotherapy are associated with improved outcomes over surgery and chemotherapy in the management of limitedstage small cell esophageal carcinoma. Radiother. Oncol. 106, 317-322. doi: 10.1016/j.radonc.2013.01.008

Nishio, M., Sugawara, S., Atagi, S., Akamatsu, H., Sakai, H., Okamoto, I., et al. (2019). Subgroup analysis of Japanese patients in a phase III study of atezolizumab in extensive-stage small-cell lung cancer (IMpower133). Clin. Lung Cancer 20, 469-476. doi: 10.1016/j.cllc.2019.07.005

O’Donnell, J. S., Madore, J., Li, X. Y., and Smyth, J. M. (2019). Tumor intrinsic and extrinsic immune functions of CD155. Semin. Cancer Biol. 65, 189-196. doi: 10.1016/j.semcancer.2019.11.013

Qu, P., Huang, X., Zhou, X., Lü, Z., Liu, F., Shi, Z., et al. (2015). Loss of CD155 expression predicts poor prognosis in hepatocellular carcinoma. Histopathology 66, 706-714. doi: 10.1111/his.12584

Scott, A. C., Dündar, F., Zumbo, P., Chandran, S. S., Klebanoff, C. A., Shakiba, M., et al. (2019). TOX is a critical regulator of tumour-specific $\mathrm{T}$ cell differentiation. Nature 571, 270-274. doi: 10.1038/s41586-019-1324-y

Sloan, K. E., Stewart, J. K., Treloar, A. F., Matthews, R. T., and Jay, G. D. (2005). CD155/PVR enhances glioma cell dispersal by regulating adhesion signaling and focal adhesion dynamics. Cancer Res. 65, 10930-10937. doi: 10.1158/0008-5472.CAN-05-1890

Song, Y., Wang, B., Song, R., Hao, Y., Wang, D., Li, Y., et al. (2018). T-cell immunoglobulin and ITIM domain contributes to CD8(+) T-cell immunosenescence. Aging Cell 17:e12716. doi: 10.1111/acel.12716

Sun, Y., Luo, J., Chen, Y., Cui, J., Lei, Y., Cui, Y., et al. (2020). Combined evaluation of the expression status of CD155 and TIGIT plays an important role in the prognosis of LUAD (lung adenocarcinoma). Int. Immunopharmacol. 80:106198. doi: 10.1016/j.intimp.2020.106198

Takai, Y., Miyoshi, J., Ikeda, W., and Ogita, H. (2008). Nectins and nectin-like molecules: roles in contact inhibition of cell movement and proliferation. Nat. Rev. Mol. Cell Biol. 9, 603-615. doi: 10.1038/ $\operatorname{nrm} 2457$

Tane, S., Maniwa, Y., Hokka, D., Tauchi, S., Nishio, W., Okita, Y., et al. (2013). The role of Necl-5 in the invasive activity of lung adenocarcinoma. Exp. Mol. Pathol. 94, 330-335. doi: 10.1016/j.yexmp,.2012.12.003

Triki, H., Charfi, S., Bouzidi, L., Ben Kridis, W., Daoud, J., Chaabane, K., et al. (2019). CD155 expression in human breast cancer: clinical significance and relevance to natural killer cell infiltration. Life Sci. 231:116543. doi: $10.1016 /$ j.lfs.2019.116543

Wang, H. H., Zaorsky, N. G., Meng, M. B., Wu, Z. Q., Zeng, X. L., Jiang, B., et al. (2015). Multimodality therapy is recommended for limited-stage combined small cell esophageal carcinoma. Onco. Targets. Ther. 8, 437-444. doi: 10.2147/OTT.S76048

Wong, A. T., Shao, M., Rineer, J., Osborn, V., Schwartz, D., and Schreiber, D. (2017). Treatment and survival outcomes of small cell carcinoma of the esophagus: an analysis of the National Cancer Data Base. Dis. Esophagus 30, 1-5. doi: 10.1111/dote. 12487

Wu, L., Mao, L., Liu, J. F., Chen, L., Yu, G. T., Yang, L. L., et al. (2019). Blockade of TIGIT/CD155 signaling reverses T-cell exhaustion and enhances antitumor capability in head and neck squamous cell carcinoma. Cancer Immunol. Res. 7, 1700-1713. doi: 10.1158/2326-6066.CIR-18-0725

Xu, L., Li, Y., Liu, X., Sun, H., Zhang, R., Zhang, J., et al. (2017). Treatment strategies and prognostic factors of limited-stage primary small cell carcinoma of the esophagus. J. Thorac. Oncol. 12, 1834-1844. doi: $10.1016 /$ j.jtho.2017.09.1966 
Xu, X., Yang, Y., Cao, L., Li, F., Zhao, J., Guo, B., et al. (1989). A retrospective study of 125 cases. Cancer Biother. Radiopharm. 34, 459-463. doi: $10.1089 / \mathrm{cbr} .2019 .2800$

Yong, H., Cheng, R., Li, X., Gao, G., Jiang, X., Cheng, H., et al. (2019). CD155 expression and its prognostic value in postoperative patients with breast cancer. Biomed. Pharmacother. 115:108884. doi: 10.1016/j.biopha.2019.108884

Zhang, Q., Bi, J., Zheng, X., Chen, Y., Wang, H., Wu, W., et al. (2018). Blockade of the checkpoint receptor TIGIT prevents NK cell exhaustion and elicits potent anti-tumor immunity. Nat. Immunol. 19, 723-732. doi: 10.1038/s41590-018-0132-0

Zhang, Y., Maksimovic, J., Naselli, G., Qian, J., Chopin, M., Blewitt, M. E., et al. (2013). Genome-wide DNA methylation analysis identifies hypomethylated genes regulated by FOXP3 in human regulatory T cells. Blood 122, 2823-2836. doi: 10.1182/blood-2013-02-481788

Zhu, Y., Qiu, B., Liu, H., Li, Q., Xiao, W., Hu, Y., et al. (2014). Primary small cell carcinoma of the esophagus: review of 64 cases from a single institution. Dis. Esophagus 27, 152-158. doi: 10.1111/dote.12069
Zou, B., Li, T., Zhou, Q., Ma, D., Chen, Y., Huang, M., et al. (2016) Adjuvant therapeutic modalities in primary small cell carcinoma of esophagus patients. A retrospective cohort study of multicenter clinical outcomes. Medicine 95:e3507. doi: 10.1097/MD.00000000000 03507

Conflict of Interest: The authors declare that the research was conducted in the absence of any commercial or financial relationships that could be construed as a potential conflict of interest.

Copyright (c) 2021 Zhao, Ma, Feng, Huang, Meng and Yu. This is an open-access article distributed under the terms of the Creative Commons Attribution License (CC BY). The use, distribution or reproduction in other forums is permitted, provided the original author(s) and the copyright owner(s) are credited and that the original publication in this journal is cited, in accordance with accepted academic practice. No use, distribution or reproduction is permitted which does not comply with these terms. 